'Hospital Sótero del Río / Pontificia Universidad Católica de Chile, Santiago, Chile.

¿Unidad de Cardiología Intervencional y Hemodinamia, Hospital Sótero del Río, Santiago, Chile.

${ }^{3}$ Clínica Universidad de los Andes, Santiago Chile.

${ }^{4}$ Clínica Alemana, Santiago Chile. aFellow Cardiología Intervencional.

Trabajo no recibió financiamiento. Los autores declaran no tener conflictos de interés.

Recibido el 28 de agosto de 2020, aceptado el 11 de noviembre de 2020

Correspondencia a:

Dr. Nicolás Veas P.

Unidad de Cardiología Intervencional y Hemodinamia Hospital Sótero del Río Avenida Concha y Toro № 3459 Puente Alto, Santiago, Chile. nicoveas@gmail.com

\section{Implante de válvula aórtica percutánea en paciente con prótesis mitral previa: riesgos y consideraciones técnicas}

\author{
RENÉ HAMEAU D. ${ }^{1, a}$, RODRIGO MUÑOZ D. ${ }^{2,4}$, \\ JOSÉ LUIS WINTER D. ${ }^{2,4}$, MARTÍN VALDEBENITO T.,2, \\ DANTE LINDEFJELD C., ${ }^{2,3}$, NICOLAS VEAS P., ${ }^{2,3}$
}

\section{Successful deployment of a percutaneous aortic valve in a patient with a mitral prosthesis. Risks and technical considerations}

The deployment of a percutaneous aortic valve is challenging in patients with a mitral prosthesis. The risk of prosthetic deformation, embolization or dysfunction is higher in this group of patients, which requires a series of technical considerations. We report a successful implantation of an Evolut Pro \# 29 self-expanding valve in a 67-year-old female with a previous Starr-Edwards caged-ball mitral prosthesis.

(Rev Med Chile 2021; 149: 286-290)

Key words: Aortic Valve Stenosis; Heart Valve Prosthesis; Heart Valve Prosthesis Implantation; Transcatheter Aortic Valve Replacement.
4 1 implante transcatéter de una válvula aórtica percutánea (TAVI) se ha convertido en una alternativa terapéutica válida frente a la cirugía cardiaca en los diversos grupos de riesgo ${ }^{1}$, especialmente en aquellos pacientes inoperables o de riesgo quirúrgico alto.

A medida que esta tecnología se vuelve accesible a un mayor número de pacientes, los cardiólogos intervencionistas se ven enfrentados a nuevos escenarios y desafíos técnicos al momento del implante. Este es el caso de aquellos pacientes con una prótesis valvular mitral previa.

Sabemos que la presencia de una prótesis mitral duplica las tasas de mortalidad de un reemplazo valvular aórtico ${ }^{2}$ y por lo tanto, muchos de estos pacientes son derivados en la actualidad para una resolución percutánea.

A pesar de que el implante de TAVI en pacientes con prótesis mitral se encuentra descrita desde el año $2008^{3}$, aún existen dudas respecto de la interacción entre ambas válvulas, las estrategias para evaluar el riesgo y el tipo de válvula preferible considerando las alternativas actuales.

A continuación presentamos el caso de una paciente con prótesis valvular mitral con jaula tipo Starr Edwards sometida al implante de TAVI transfemoral.

\section{Caso clínico}

Presentamos el caso de una paciente de sexo femenino, 67 años, con antecedentes mórbidos de diabetes mellitus tipo 2, hipertensión arterial, hipotiroidismo, fibrilación auricular (FA) y enfermedad reumática inactiva, siendo sometida a un reemplazo valvular mitral con una prótesis con jaula ("caged-ball") tipo Starr Edwards el año 1996.

Ingresa por cuadro disnea y deterioro progresivo de su capacidad funcional hasta WHO III hace 12 meses. ECG de ingreso mostraba hemibloqueo izquierdo anterior y una FA lenta a 50 latidos por minuto (lpm). 
Se solicitó un ecocardiograma transtorácico (EcoTT) que mostró un ventrículo izquierdo (VI) de tamaño normal y con función sistólica preservada (FEVI 58\%), prótesis mitral normofuncionante y válvula aórtica de 3 velos engrosados con signos de estenosis severa e insuficiencia leve (gradiente medio de $41 \mathrm{mmHg}$, velocidad máxima $4 \mathrm{~m} / \mathrm{seg}$, área valvular $0,8 \mathrm{~cm}^{2}$ ), Presión sistólica de arteria pulmonar (PSAP) $52 \mathrm{mmHg}$.

Para completar el estudio, se realiza una coronariografía que no mostró lesiones coronarias significativas.

Se decide discutir el caso en Heart Team, dado el riesgo inherente de una reoperación y por otro lado, las dificultades adicionales que implica el implante de válvula aórtica percutánea (TAVI) en pacientes con prótesis mitral con jaula. El riesgo de mortalidad por score STS -PROM era de 7,64\% y de $22,2 \%$ para morbi-mortalidad. El riesgo de mortalidad intrahospitalaria post TAVI por score STS/ACC era de $2,13 \%$.

Tras una discusión con el paciente y sus familiares, se decide el implante de TAVI, completando el estudio de laboratorio e imagenológico con AngioTC cardiaco gatillado según protocolo (Figura 1). Este demostró un anillo aórtico calcificado con un perímetro de $77,9 \mathrm{~mm}$, diámetro de $24,8 \mathrm{~mm}$ y un área estimada en $468 \mathrm{~mm}^{2}$. La dis- tancia entre la prótesis mitral y el plano del anillo aórtico era de 6,22 $\mathrm{mm}$. El ángulo entre el plano mitral y el tracto de salida del VI era cercano a $35^{\circ}$. Estas mediciones fueron compatibles con válvula Evolut Pro \#29 (Medtronic Inc., Minneapolis, $\mathrm{MN})$. Ambos accesos femorales se consideraron adecuados para el implante.

Al momento del implante, utilizando sedación consciente según protocolo local ${ }^{4}$, se instala una vaina $6 \mathrm{~F}$ en arteria femoral izquierda para el avance de un pigtail hacia el plano valvular y una vaina venosa femoral izquierda para posicionar una sonda marcapasos transitoria (SMPT) en el ventrículo derecho (Arrow ${ }^{\otimes}$ Temporary Pacing Catheter Kit).

Utilizamos la arteria femoral derecha para el implante de la TAVI con un introductor Sentrant 16F. A continuación, se cruza válvula aórtica con cateter JR4 5F (Optitorque, Terumo ${ }^{\circledR}$ ) con una guía recta (Medtronic Inc., Minneapolis, MN), intercambiando a guía J 0,035 (Medtronic Inc., Minneapolis, MN). Se realizan diversas proyecciones para confirmar que no interfiera con la válvula mitral y luego, se avanza un pigtail $6 \mathrm{~F}$ (Medtronic Inc., Minneapolis, MN) hacia el VI. Posteriormente, se intercambia por una guía de alto soporte Confida (Medtronic Inc., Minneapolis, MN) y se avanza la válvula Evolut Pro \#29 que

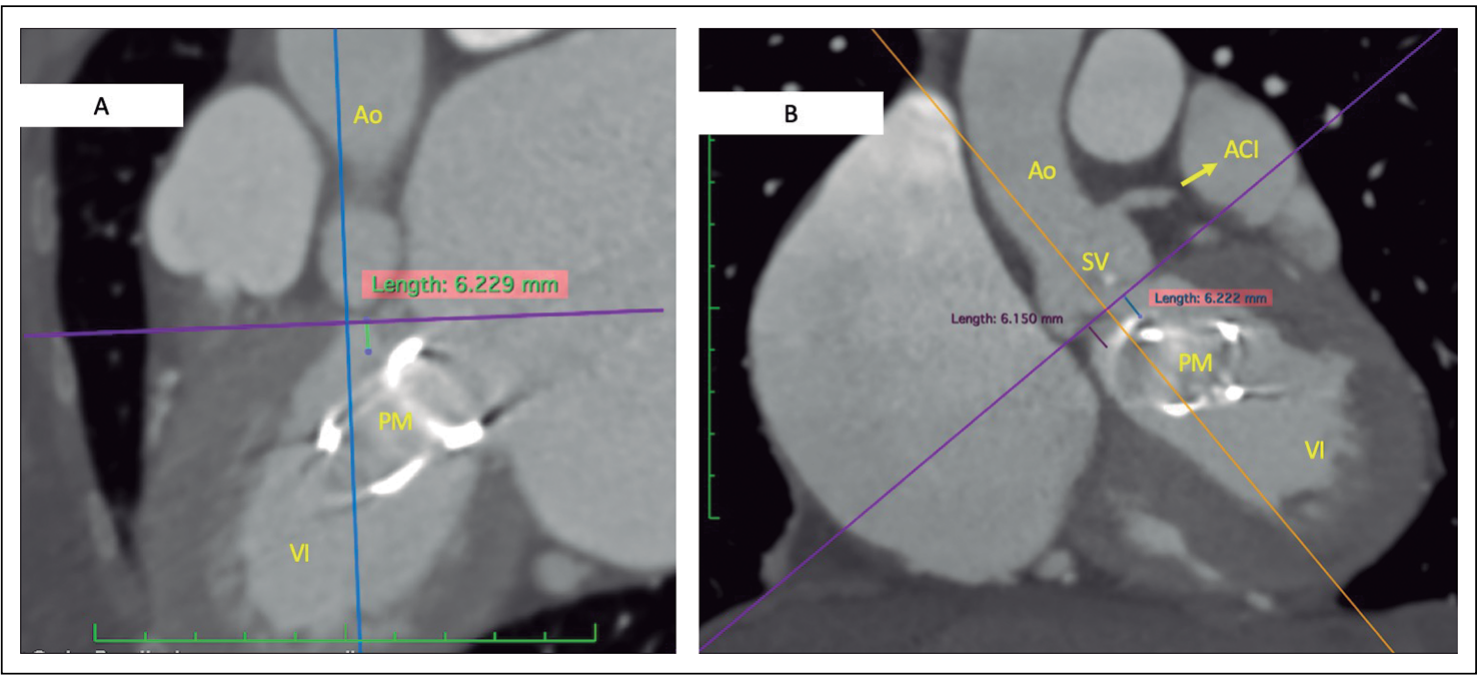

Figura 1. Evaluación preoperatoria con AngioTC. Uso del AngioTC para evaluar la distancia entre el anillo aórtico y el plano valvular mitral. VI: Ventrículo izquierdo, Ao: Aorta ascendente, SV: Senos de Valsalva, PM: Prótesis Mitral, ACl: Arteria coronaria izquierda. 


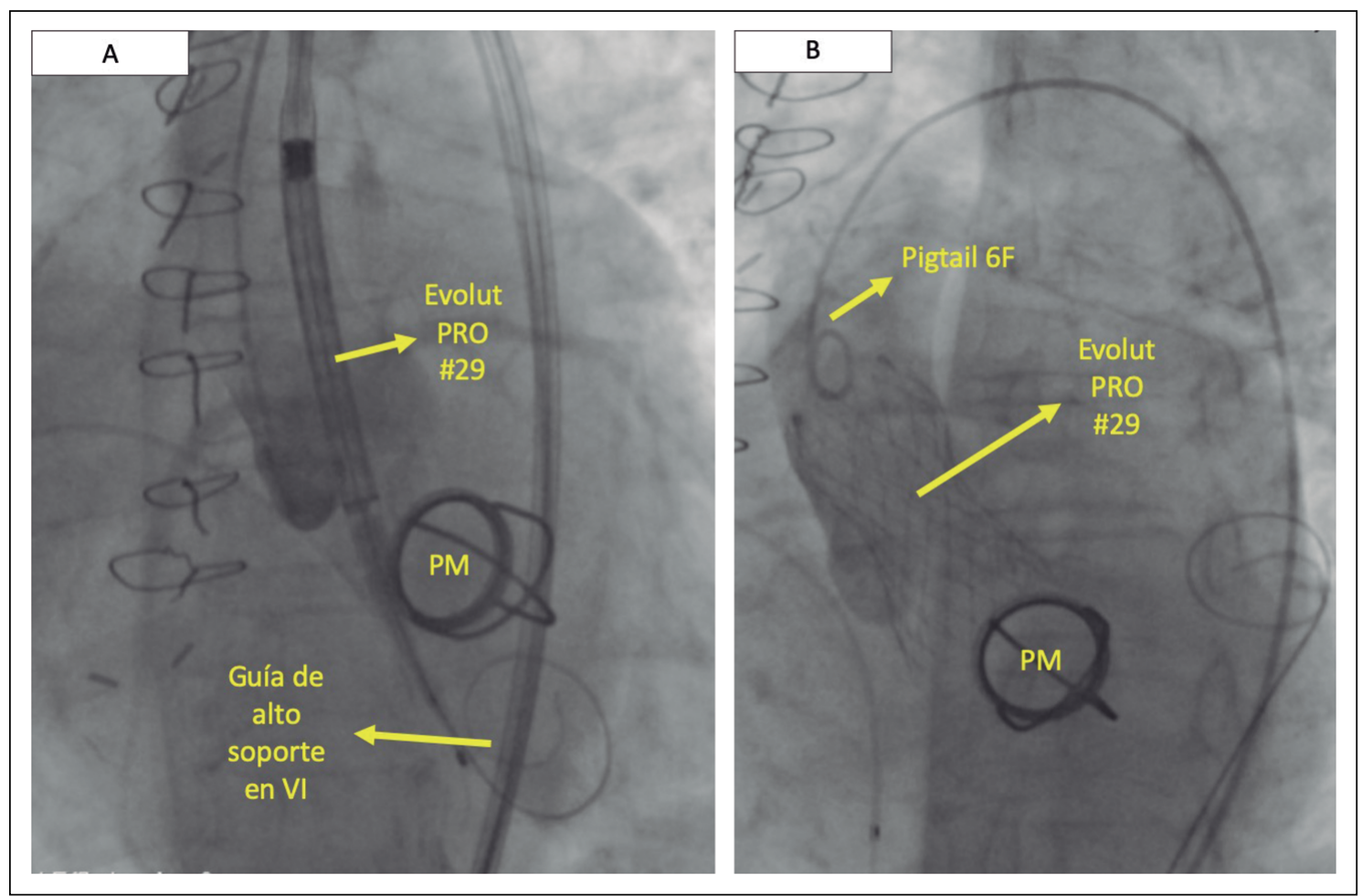

Figura 2. Imágenes fluoroscópicas de la intervención. A) Posicionamiento de una guía de alto soporte en el ventrículo izquierdo (VI) y luego avance de la TAVI. El punto fundamental es corroborar en diversas proyecciones que no exista interacción entre esta y la prótesis mitral (PM). B) Resultado final con la válvula autoexpandible desplegada a nivel del plano aórtico.

se despliega lentamente, asegurando en todo momento la indemnidad de la prótesis Starr Edwards (Figura 2). Se decide una liberación controlada de la TAVI con marcapaso a $140 \mathrm{lpm}$ para asegurar la posición. En este caso, fue necesaria la recaptura en una ocasión para lograr el despliegue a una profundidad que no interfiriera con el funcionamiento valvular mitral.

El procedimiento se lleva a cabo sin incidentes, comprobándose por ecocardiograma TT, una prótesis aórtica normofuncionante con gradiente máximo de $8 \mathrm{mmHg}$ y mínimo reflujo. La prótesis mitral no presentaba alteraciones funcionales. Cierre del acceso femoral con 2 Perclose Proglide (Abott ${ }^{\circledR}$, Chicago, Illinois, EEUU).

La paciente persistió con FA bloqueada a las $48 \mathrm{~h}$ post implante por lo que se implantó marcapasos VVI, siendo dada de alta en buenas condiciones generales a las $72 \mathrm{~h}$ y sin requerimiendos de Unidad Crítica. El procedimiento y su posterior publicación contó en el consentimiento informado de la paciente y se siguieron todas las normas locales así como las recomendaciones de la declaración de Helsinski (2013).

\section{Discusión}

Reportamos un caso de implante de válvula aórtica percutánea en una paciente con una prótesis valvular mitral con jaula previa.

Sabemos que la existencia de una prótesis mitral conlleva potenciales dificultades al momento del implante. Entre estas podemos destacar: 1) Riesgo teórico de deformar la prótesis mitral en caso de desplegar una válvula con mucha fuerza radial o, más probablemente, el alterar la arquitectura del marco de Nitinol de la TAVI dada la mayor rigidez del marco prótesico mitral; 2) Riesgo potencial de cruzar a través de la prótesis mitral con la guía previo al implante; 3) Otro factor a considerar es la posible interferencia de la TAVI con la esfera protésica y generar una insuficien- 
cia mitral severa; 4) Dada la estrecha relación de ambas válvulas a nivel de la ventana mitroaórtica, existe el riesgo de generar obstrucción del tracto de salida del VI; 5) Riesgo de embolización de la prótesis aórtica al momento de su despliegue (efecto "watermelon seed").

Otro aspecto relevante a considerar es la utilidad que presta la angiotomografía computada en la planificación de estos procedimientos. Esta nos permite evaluar el ángulo entre la posición mitral y el tracto de salida del VI, así como medir la distancia entre el anillo aórtico y la estructura metálica protésica, lo cual se ha descrito como un predictor de embolización. En caso de que existan estos predictores, algunos autores sugieren, por ejemplo, el uso de la balonplastía aórtica previa para evaluar el potencial comportamiento de la TAVI durante su despliegue definitivo. Esto también nos puede llevar a ser más conservadores a la hora de elegir el diámetro final que queremos alcanzar con la TAVI (evitando la sobredimensión de la válvula u "oversizing") o a evitar la postdilatación agresiva para el cierre de leaks con el objeto de evitar malposicionamiento o embolización de esta ${ }^{5}$.

Teniendo en cuenta estas consideraciones técnicas, se optó por el uso de una válvula autoexpandible que constituye el tipo de TAVI más frecuentemente utilizado en la literatura para este escenario. Las principales ventajas radican en su posición supraanular y por otro lado, la posibilidad de recapturarse en caso de generar interferencia con la prótesis mitral ${ }^{6}$.

Por último, recomendamos la utilización de marcapaso ventricular rápido al momento de la liberación para lograr un posicionamiento más preciso.

El manejo perioperatorio también presenta desafíos dada la alta prevalencia del uso de anticoagulantes en este grupo; factor que aumenta el riesgo de complicaciones hemorrágicas y que constituyen un predictor independiente de mortalidad en este grupo.

En 2017, Amat Santos et al ${ }^{7}$, publicó los resultados de un estudio multicéntrico de 91 pacientes (total: 2414) con o sin prótesis mitral (PM) sometidos a TAVI. Cabe destacar que en esta serie, un 73,6\% $(\mathrm{n}=67)$ tenían una prótesis mitral mecánica ( $19,4 \%$ monodisco y $80,6 \%$ bidisco), no existiendo pacientes con prótesis con jaula como nuestro caso. Entre la principales conclusiones podemos destacar que no hubo diferencias en mortalidad vs aquellos sin PM. Sin embargo, se observó un mayor riesgo de embolización del dispositivo $(6,7 \%$ vs $3,3 \%)$ en aquellos con una distancia PM-anillo aórtico $<7 \mathrm{~mm}$ y además un mayor riesgo hemorrágico en el grupo con PM $(24,2 \%$ vs $16,1 \%$; p 0,041), incluso en aquellos con acceso transfemoral. Los principales predictores de mortalidad en este grupo fueron los eventos hemorrágicos, fibrilación auricular previa, clase funcional NYHA y la enfermedad pulmonar obtructiva crónica.

Respecto de las prótesis mitral con jaula ("caged-ball”) , los primeros casos publicados de TAVI en este escenario se remontan al año 2013 por Gedilki et $\mathrm{al}^{8}$ y Rahman et $\mathrm{al}^{9}$. En ámbito nacional, Maluenda et $\mathrm{al}^{10}$, también reporta en $2016 \mathrm{el}$ implante exitoso de una TAVI autoexpandible y recientemente, Eftychiou et $\mathrm{al}^{11}$ lo hace con una serie de 2 pacientes pero utilizando prótesis balón expandibles. En su conjunto, estas experiencias muestran buenos resultados perioperatorios y asusencia de complicaciones mayores, pero refuerzan la necesidad de una adecuada planificación del caso.

En conclusión, el implante de TAVI en presencia de una prótesis mitral es un escenario desafiante que requiere de una rigurosa planificación para anticiparse a los riesgos potenciales y lograr un resultado exitoso.

\section{Referencias}

1. Popma J, Deeb G, Yakubov S MM, Gada H, OHair D, et al. Transcatheter Aortic-Valve Replacement with a Self-Expanding Valve in Low-Risk Patients. N Engl J Med. 2019; 380: 1706-15.

2. Lung B, Baron G, Butchart E DF, Gohlke-Bärwolf C, Levang $\mathrm{O}$, et al. A prospective survey of patients with valvular heart disease in Europe: The Euro Heart Survey on valvular heart disease. Eur Heart J. 2003; 24 (1): 1231-43.

3. Rodés-Cabau J, Dumont E, Miró S, Doyle D, De Larochellière $\mathrm{R}$, Clavel $\mathrm{M}$ et al. Apical aortic valve implantation in a patient with a mechanical valve prosthesis in mitral position. Circ Cardiovasc Interv. 2008; 1 (1): 233-5.

4. Hameau R, Veas N, Winter J, Valdebenito M, Muñoz R, Fuensalida A, et al. Enfoque minimalista en el implante de válvula aórtica percutánea. Rev Chil Cardiol. 2019; 38 (3): 173-81. 
5. Zacharoulis A, Antoniades A, Frogoudaki A, Kolokathis F, Lekakis J et al. TAVR in patients with aortic stenosis and mechanical mitral valve. Int J Cardiol. 2015; 180 (1): 226-7.

6. Moon S, Ko Y, Hong G, Lee S, Chang B, Shim J, et al. Transcatheter aortic valve implantation in a patient with previous mitral valve replacement. Korean Circ J. 2014; 44 (5): 344-7. doi:10.4070/kcj.2014.44.5.344

7. Amat-Santos I, Cortés C, Nombela L, Muñoz-García A, Suárez De Lezo J, Gutiérrez-Ibañes E, et al. Prosthetic Mitral Surgical Valve in Transcatheter Aortic Valve Replacement Recipients: A Multicenter Analysis. JACC Cardiovasc Interv. 2017; 10 (19): 1873-1981.

8. Gedikli O, Kutlu M, Civelek A. Transcatheter implan- tation of a CoreValve aortic prosthesis in a patient with a ball-cage mechanical mitral valve. J Hear Valve Dis. 2013; 22 (1): 697-700.

9. Rahman MS, Roy D, Brecker SJ. Transcatheter aortic valve implantation in a patient with previous Starr-Edwards mitral valve prosthesis. Heart. 2013; 99 (1): 217-8.

10. Maluenda G, Caorsi C, Baeza C. Transfemoral implantation of CoreValve Evolut-R aortic prosthesis in patient with prior ball-cage mechanical mitral valve prosthesis. Cardiovasc Revascularization Med. 2016; 17 (1): 287-9.

11. Eftychiou C, Kokkinos C, Houri S. TAVI with Balloon Expandable Aortic Valve Prosthesis in the Presence of Mechanical Mitral Valve Prosthesis. EC Cardiol. 2019; 6.6 (1): 536-42. 